\title{
Efecto de la amigdalotomía e hipotalamotomía unilateral en pacientes con agresividad refractaria
}

\author{
Luis García-Muñoz ${ }^{1,2}$, Ofir Picazo-Picazo ${ }^{3}$, José D. Carrillo-Ruíz²,4, Jesús Favila-Bojórquez ${ }^{5,6}$, \\ Fernando Corona-García ${ }^{7}$, Miguel Ángel Meza-Bautista ${ }^{8}$ y Fiacro Jiménez-Ponce ${ }^{*}$ \\ ${ }^{1}$ Servicio de Neurología y Neurocirugía, Hospital General de México "Dr. Eduardo Liceaga"; ${ }^{2}$ Servicio de Neurocirugía y Neurología, Hospital de \\ Especialidades Centro Médico Nacional Siglo XXI, IMSS, Ciudad de México; ${ }^{3}$ Escuela Superior de Medicina, Instituto Politécnico Nacional, Ciudad \\ de México; ${ }^{4}$ Coordinación de Neurociencias, Universidad Anáhuac, Ciudad de México; ${ }^{5}$ Servicio de Psiquiatría, Hospital de Especialidades, Centro \\ Médico Nacional Siglo XXI, IMSS, Ciudad de México; ${ }^{6}$ Higiene Mental, Secretaría de Salud, Ciudad de México; ${ }^{7}$ Servicio de hospitalización, Hospital \\ Psiquiátrico Fray Bernardino Álvarez, Secretaría de Salud, Ciudad de México; ${ }^{8}$ Servicio de Neurocirugía, Hospital Regional 110, IMSS, Guadalajara, \\ Jalisco; ${ }^{9}$ Subdirección de Regulación y Atención Hospitalaria, Instituto de Seguridad y Servicios Sociales de los Trabajadores del Estado, Ciudad \\ de México. México
}

\section{Resumen}

Antecedentes: El tratamiento neuroquirúrgico, aunque polémico, se considera un recurso útil en el tratamiento de enfermedades psiquiátricas crónicas como la agresividad refractaria. Objetivo: Evaluar los resultados clínicos y los efectos colaterales de la hipotalamotomía posteromedial (HPM) asociada a amigdalotomía en pacientes con agresividad refractaria. Método: Se realizó un ensayo clínico en pacientes con agresividad crónica y refractaria a tratamiento farmacológico. Se les realizó amigdalotomía central asociada a HPM mediante termocoagulación por radiofrecuencia. El grado de agresividad se cuantificó mediante la escala global de agresividad de Yudofsky. Los cambios postoperatorios en la conducta agresiva continuaron siendo evaluados cada 6 meses durante al menos 36 meses. Resultados: Se observó un cambio estadísticamente significativo de la conducta agresiva, a lo largo de 36 meses de seguimiento. Se describen los efectos colaterales de la asociación de ambos procedimientos, siendo el de mayor frecuencia la somnolencia y algunos casos de reducción en la conducta sexual. Conclusión: Las lesiones unilaterales simétricas y simultáneas del núcleo central de la amígdala y del hipotálamo posteromedial contralaterales a la dominancia motora dan el mismo efecto clínico en la reducción de la agresividad patológica que las lesiones bilaterales.

PALABRAS CLAVE: Sistema Límbico. Agresividad. Escala Global de Agresividad. Neurocirugía Estereotáxica. Psicocirugía.

\begin{abstract}
Background: Neurosurgical treatment, although controversial, is considered a useful resource in the treatment of chronic psychiatric diseases such as refractory aggressiveness. Objective: To evaluate the clinical results and side effects of posteromedial hypothalamotomy associated with amygdalotomy in patients with refractory aggressiveness. Method: $A$ clinical trial was conducted in patients with chronic aggressiveness and refractory to pharmacological treatment. A central amygdalotomy associated with posteromedial hypothalamotomy was performed using thermo-coagulation by radiofrequency. The degree of aggressiveness was quantified by the Yudofsky's global scale of aggression. Postoperative changes in aggressive behavior continued to be evaluated every 6 months for at least 36 months. Results: A statistically significant change in aggressive behavior was observed during 36 months of follow-up. The collateral effects of the association of both procedures are described, the most frequent being drowsiness and some cases of reduction in sexual behavior. Conclusion: Symmetric and simultaneous unilateral lesions of the central nucleus of the amygdala and the posteromedial hypothalamus contralateral to motor dominance give the same clinical effect in the reduction of the pathological aggression that the bilateral lesions.
\end{abstract}

KEY WORDS: Limbic System. Aggressiveness. Global Aggressiveness Scale. Stereotactic Neurosurgery. Psychosurgery.

Correspondencia:

*Fiacro Jiménez-Ponce

E-mail: fiacrojimenez@yahoo.com
Fecha de recepción: 06-03-2019

Fecha de aceptación: 22-03-2019

DOI: 10.24875/GMM.19005128
Gac Med Mex. 2019;155(Suppl 1):S62-S69

Disponible en PubMed

www.gacetamedicademexico.com 


\section{Antecedentes}

La conducta agresiva «normal» tiene objetivos muy específicos: mantener la integridad física del individuo, depredación con fines de subsistencia, la conservación del espacio vital o territorio y la perpetuación de la especie. Estas funciones se resumen perfectamente en el término expresado por Walter Canon', fight or flight. La agresividad humana muestra un patrón común de conductas similares a las otras especies animales y solo se activan ante un estímulo amenazante. Esta conducta innata se encuentra asociada con el sistema límbico. La primera descripción de la existencia de un "cerebro emocional» fue hecha por Christfried Jakob² en 1907-1908, estableciendo las bases para las contribuciones posteriores de James W. Papez en 1932 y de Paul D. MacLean'. Estos conceptos culminaron en la integración del sistema límbico y dieron un mejor entendimiento, entre la fisiología y la anatomía, en la generación y control de los patrones de conducta y emociones. Por otro lado, la agresividad «no natural o patológica» es una conducta exagerada que se presenta de manera desproporcionada ante un estímulo que normalmente no implicaría peligro. Este síntoma se acompaña con cierta frecuencia en algunas enfermedades neurológicas y/o psiquiátricas. De acuerdo con Yudofsky la conducta agresiva patológica es estereotipada y se puede manifestar emitiendo ruidos o lenguaje soez, produciendo daño a objetos o animales, infringiendo lesiones físicas a otras personas (heteroagresividad) o contra ellos mismos (autoagresividad) ${ }^{3}$. Yudofsky considera que la conducta agresiva en pacientes está relacionada con daño neurológico o psiquiátrico, por lo que debe ser considerado como un «trastorno neuroagresivo". El tratamiento de este trastorno implica el uso de fármacos neurolépticos, benzodiacepinas, antiepilépticos, antidepresivos, betabloqueadores o moduladores del estado de ánimo ${ }^{4,5}$. Los esquemas farmacológicos siempre se administran combinados y por tiempo prolongado. Sin embargo, existe una «refractariedad" al tratamiento farmacológico que para motivos de este trabajo se considera como una respuesta insuficiente o nula al mismo. Así, la terapia electroconvulsiva (TEC) se propone como un recurso terapéutico más.

El trastorno neuroagresivo refractario es un problema no solo por el riesgo social que implica, sino también por los altos costos del tratamiento farmacológico, las frecuentes hospitalizaciones que se requieren para el control de las crisis y los cuidados de «contención» que los familiares deben proporcionar al paciente.

Gotlieb Burckhardt fue quién en el siglo XIX lesionó quirúrgicamente por primera vez los lóbulos frontales o temporales en seis pacientes psiquiátricos con resultados parciales y con alta morbimortalidad ${ }^{6}$. Ya en el siglo XX Fulton resecó la corteza frontal en simios transformando así la conducta normalmente irritable a dócil'. Estos resultados motivaron a Lima y Moniz (en Portugal) y a Freeman y Watts (en Norteamérica) a efectuar una sección neuroquirúrgica de las conexiones frontales hacia los núcleos basales en pacientes psiquiátricos. Sin embargo, a pesar de los resultados en muchos acasos alentadores, este procedimiento denominado lobotomía frontal no mostraban una metodología clara, no establecían las indicaciones precisas y presentaban una alta morbilidad, motivo por el cual cayó en el descredito, ${ }^{1,-10}$.

Paralelamente la aparición simultánea de técnicas quirúrgicas sofisticadas de localización, el desarrollo de atlas cerebrales estereotáxicos y de los métodos de rayos $X$ facilitaron establecer una relación entre la funcionalidad de las estructuras límbicas y las conductas derivadas de ellas. Así, algunas estructuras anatómicas del sistema límbico se convirtieron en blancos quirúrgicos para el tratamiento selectivo de la conducta agresiva'.

Específicamente la hipotalamotomía y la amigdalotomía se propusieron separadamente para el tratamiento de la conducta agresiva ${ }^{7-10}$. El hipotálamo posteromedial fue lesionado bilateralmente por Sano, et al. en 1966, quienes reportaron una reducción significativa de la conducta agresiva ${ }^{10,11}$. Por otro lado, Ramamurthi y Balasubramanian reportaron también una reducción importante de la conducta agresiva humana mediante lesión bilateral del núcleo central de la amígdala, confirmando los resultados de Narabayashi' ${ }^{12,13}$. Ambos procedimientos han demostrado ser efectivos para el control de la conducta agresiva refractaria. Sin embargo, la hipotalamotomía posteromedial (HPM) bilateral puede producir una disfunción hipotalámica grave y potencialmente fatal, ya Sano mismo había recomendado un periodo de espera entre 7 y 10 días entre la realización de ambas lesiones ${ }^{11}$. Para la amigdalotomía central (AC) bilateral, el daño funcional se manifiesta con el síndrome de Klüver-Bucy (agnosia, visual estímulos potencialmente peligrosos, hiperoralidad e hipersexualidad, entre otros síntomas) ${ }^{14}$. Andy y Stephan publicaron resultados al respecto de que la combinación de lesiones de la amígdala y el hipotálamo no aumentaron el 
porcentaje de mejoría de los resultados obtenidos por la sola $\mathrm{AC}^{15}$.

Por otro lado, ya en 2012 la combinación de la cingulotomía y capsulotomía anterior bilaterales fueron propuestas para el control de la conducta agresiva refractaria ${ }^{16}$.

Como alternativa terapéutica sustituta de la lesión por radiofrecuencia, Franzzini en el 2013 trató pacientes con agresividad refractaria empleando estimulación eléctrica cerebral profunda en ambos hipotálamos posteriomediales (HPM) ${ }^{17}$. Sin embargo, el procedimiento está aún en fase de investigación.

Ya nuestro grupo ha publicado los resultados históricos preliminares de una serie de casos en los que la lesión unilateral simétrica de la AC y de la HPM contralateral a la dominancia manual disminuyeron la conducta agresiva refractaria en el trastorno neuroagresivo ${ }^{18}$.

Así, los objetivos principales del presente ensayo clínico fueron evaluar los resultados clínicos y los efectos colaterales de la HPM asociada a AC, de manera unilateral, simétricas o asimétricas, ipsilaterales o contralaterales a la dominancia manual.

\section{Método}

Entre los años 2012 y 2017 se trató a pacientes con el diagnóstico de agresividad refractaria que fueron referidos por médicos psiquiatras de diferentes instituciones y quienes solicitaron manejo neuroquirúrgico para el tratamiento de la agresividad refractaria. Todos los casos se presentaron a un comité integrado por dos psiquiatras y dos neurocirujanos funcionales. Como criterios de inclusión se establecieron los siguientes requisitos: a) cronicidad del cuadro clínico manifestada durante al menos 5 años de evolución; b) dificultad para el control de la conducta agresiva (llevando un tratamiento farmacológico correcto, durante al menos dos años y con buena adherencia terapéutica) 0 , que a pesar de tener una respuesta clínica adecuada, se manifestaran efectos adversos por la medicación empleada como disfunción hepática, diabetes mellitus o síndrome metabólico, y c) estudios de neuroimagen que descartaran enfermedad neoplásica, vascular, infecciosa, desmielinizante o parasitaria. Los pacientes que fueron tratados con TEC no fueron rechazados para manejo quirúrgico. En todos los casos se descartaron discrasias sanguíneas, se determinó el riesgo cardiovascular cuando fue necesario y se les realizó resonancia magnética cerebral, tanto para descartar enfermedades agregadas como para planeación quirúrgica estereotáxica. Se solicitó el consentimiento bajo información para realizar la AC y la HPM mediante termocoagulación por radiofrecuencia (TCRF), este se obtuvo de forma directa cuando los pacientes tenían autonomía y capacidad para decidirlo o por medio de los padres o tutores. Para medir y determinar la conducta agresiva se empleó la Escala Explícita de Agresividad propuesta por Yudofsky o mejor conocida como Over Aggression Scale (OAS), que es un instrumento de medición validado en México ${ }^{19}$. La escala contempla cuatro capítulos, los cuales consisten en agresividad verbal, agresividad contra objetos, autoagresividad y heteroagresividad. Cada capítulo tiene un valor máximo de 4 puntos y la calificación total es de 16. La determinación de la dominancia motora se realizó con la escala de Edinburgo ${ }^{20}$.

Por la naturaleza del padecimiento, los pacientes se hospitalizaron un día antes del procedimiento quirúrgico, manteniéndose su tratamiento farmacológico tal como estaba indicado por el médico psiquiatra. El procedimiento quirúrgico se efectuó bajo anestesia general, así se colocó el marco estereotáxico y se realizó tomografía craneal computarizada para la planeación de los sitios anatómicos. Para localizar la AC y el HPM se utilizaron o el sistema estereotáxico Zamorano-Dujovny (Z-D) (Fischer, Leibinger, Alemania) o el marco Leksell (Elekta AB, Suecia). Para localizar la HPM se tomaron como referencia las coordenadas estereotáxicas reportadas por Sano: $\mathrm{x}=2.0 \mathrm{~mm}$ lateral a la pared del tercer ventrículo, $y=$ punto medio comisural y $z=2.0 \mathrm{~mm}$ debajo de la línea intercomisural. La coordenada z se exploró desde los $4 \mathrm{~mm}$ arriba del sitio para iniciar la estimulación eléctrica previa a la lesión ${ }^{11}$. Para la localización de la AC, se tomó como referencia las coordenadas de Andy y Stephan ${ }^{18}$, las cuales se ajustaron con el Atlas Cerebral Estereotáxico de Talairach ${ }^{21}$ y de Schaltenbrand y Warrhen: $x=20-26$ (24) mm para el componente lateral de la amígdala, y = 3 a 5 (2 a 4) mm en frente del cuerno temporal y z a $7 \mathrm{~mm}$ por arriba del piso del ventrículo temporal (o $15 \mathrm{~mm}$ por debajo de la línea intercomisural). Para el cálculo de coordenadas en las imágenes de los pacientes se emplearon los programas Stereoplan ${ }^{\circledR}$ (Fischer, Leibinger. Alemania) o Praezis ${ }^{\circledR}$ (Leibinger, Alemania) o el programa Framelink $^{\circledast}$ (Medtronic, EE.UU.). Para abordar los núcleos se realizó un trépano coronal a $3 \mathrm{~cm}$ por fuera de la línea media.

Ante las complicaciones funcionales reportadas en la literatura médica al realizar lesiones bilaterales del 
mismo núcleo y al mismo tiempo, tanto para el complejo amigdalino (NCA) como del hipotálamo posteromedial, nosotros combinamos los núcleos, ya fuera de manera simultánea unilateral para ambos o bilateral en tiempos diferentes.

La lesión del NCA se realizó con un electrodo bipolar de $0.5 \mathrm{~mm}$ de diámetro (Fischer, Leibinger, Alemania), a $80{ }^{\circ} \mathrm{C}$ durante 90 segundos. Para la lesión hipotalámica se empleó un electrodo bipolar de $0.3 \mathrm{~mm}$ de diámetro (Fischer, Leibinger, Alemania), a $80^{\circ} \mathrm{C}$ durante 60 segundos, empleando un generador de lesiones por TCRF ( $\mathrm{N}-50$, Fischer, Leibinger, Alemania). Solo para la HPM, se realizaron pruebas de estimulación eléctrica empleando voltajes entre $1.0 \mathrm{y}$ $8.0 \mathrm{~V}, 500-1000 \mu \mathrm{s}$ y $100 \mathrm{~Hz}$ de manera continua hasta obtener una respuesta simpática. La correcta ubicación del electrodo dentro del HPM provocó midriasis ipsilateral, hipertensión arterial y taquicardia.

Cuando se realizó la lesión por TCRF tanto de la AC como del HPM en el mismo tiempo quirúrgico, se denominó al procedimiento como simultáneo. Cuando se realizaron las lesiones de ambos núcleos en el mismo hemisferio cerebral, se le denominó simétrico. Cuando los núcleos tratados no estuvieron en el mismo hemisferio cerebral, por ejemplo, AC derecha e HPM izquierda o viceversa, se denominó asimétrico. Si solo se operó un núcleo, AC o HPM, se denominó como unilateral. En esta serie no se realizó lesión por TCRF bilateral simultánea, ni de la AC ni del HPM para evitar daño funcional por lesión bilateral.

Los cambios postoperatorios en la conducta agresiva continuaron siendo evaluados cada 6 meses durante al menos 36 meses con la escala OAS.

\section{Resultados}

El estudio incluyó 12 pacientes, 11 hombres y 1 mujer. La media de la edad fue de $31.8 \pm 11.48$ desviación estándar (DE). La agresividad patológica refractaria estuvo presente en ocho casos con retraso mental (RM), de este grupo cuatro sujetos no desarrollaron lenguaje, dos se relacionaron con síndrome de Asperger, uno con autismo y otro con epilepsia. Dos sujetos se relacionaron con esquizofrenia, uno con síndrome postraumático (que además desarrolló trastorno obsesivo-compulsivo) y otro con trastorno límite de la personalidad. Todos los pacientes mostraron un patrón de agresividad extrema de 16 puntos, lo cual es el máximo posible de acuerdo con la escala global de Yodosfsky2. Cuatro casos mostraron heteroagresividad y ocho de forma combinada (heteroagresividad más autoagresividad). Cinco pacientes masculinos mostraron conducta sexual aumentada y desinhibida, manifestada con masturbación en público y un caso se acompañó además de acoso sexual. En cuatros casos no se reportó la conducta sexual y en tres se mencionó como «normal». Nueve pacientes fueron diestros. Todos los pacientes recibieron al menos un neuroléptico (quetiapina, haloperidol, ziprasidona, olanzapina, risperidona, cloropromazina y clozapina) asociado a un antiepiléptico (carbamazepina, valproato de Mg, clonazepam, primidona, levetiracetam y lorazepam). En un caso se utilizó metilfenidato. Cada paciente recibió entre tres y cinco fármacos para reducir la conducta agresiva y en todos los casos a excepción de uno, se presentaron efectos adversos, los más frecuentes fueron los fenómenos extrapiramidales (distonía de torsión) en seis casos, diabetes mellitus en cuatro pacientes, un síndrome metabólico con plaquetopenia y ginecomastia y otro con somnolencia y sialorrea. Nueve pacientes recibieron TEC previo a la cirugía (Tabla 1).

En los primeros cinco casos se seleccionó al azar una TCRF unilateral tanto de un NCA y de un HPM de forma simultánea pero no de manera simétrica. Estos primeros casos tratados tuvieron que ser reintervenidos en menos de tres meses debido a la recidiva de la conducta agresiva. Con el segundo procedimiento quirúrgico se pudo observar que al completar la bilateralidad de los núcleos, ya fuera de la amígdala o del hipotálamo posteromedial se lograba el efecto de terapéutico siempre y cuando las lesiones del HPM y del NCA coincidieran contralaterales a la dominancia manual. Las observaciones y el análisis de estos primeros cinco casos reafirmaron los datos del estudio previo sobre la dominancia del HPM y del NCA. En los siete pacientes subsecuentes se realizó la asignación del sitio en función de la dominancia motora (Tabla 2).

Con fines de este reporte, se realizó seguimiento desde los $6,12,18,24,30$ y 36 meses. Los primeros nueve casos ya han alcanzado los 60 meses de seguimiento.

En la tabla 3 se observan los cambios de los valores obtenidos en la OAS. Aplicando la prueba no paramétrica de rangos de Wilcoxon de las medidas obtenidas en el periodo basal en comparación con cada seguimiento se observó un cambio estadísticamente significativo, no obstante que no se completó el seguimiento a 36 meses en los últimos tres casos.

En la figura 1 se aprecian las imágenes de resonancia magnética típicas del postoperatorio inmediato a la realización de la AC y la HPM. Los principales efectos secundarios y los cambios en la conducta 
Tabla 1. En esta tabla se observan los datos demográficos de los doce pacientes sometidos a amigdalotomía e hipotalamotomía posteromedial

\begin{tabular}{|c|c|c|c|c|c|c|c|c|c|}
\hline Caso & Sexo & Edad & Comorbilidad & Lenguaje & Sexualidad & Tipo & Dominancia & Medicación & Efectos Adversos \\
\hline LSF & M & 28 & $\mathrm{RM}$ & Si & Hiper & Hetero & Diestro & QTP, HPD, ZSD, CBZ & Distonías de torsión \\
\hline ASF & M & 22 & $\mathrm{RM}+$ Asperger & No & Hiper & Hetero/Auto & Zurdo & OLZ, CBZ, VPM, CLZ & Distonías de torsión \\
\hline AAJ & M & 23 & RM+Autismo & No & NR & Hetero/Auto & Diestro & QTP, HPD, ZSD, CBZ & Diabetes mellitus \\
\hline $\mathrm{RCA}$ & M & 28 & $\mathrm{RM}$ & $\mathrm{Si}$ & Hiper & Hetero & Diestro & VPM, LTC, CLZ, QTP, LMZ & Diabetes mellitus \\
\hline NHR & M & 33 & $\mathrm{RM}$ & No & Hiper & Hetero/Auto & Zurdo & $\begin{array}{l}\text { HPD, LTC, PMD, CLZ, } \\
\text { LMZ }\end{array}$ & $\begin{array}{l}\text { Diabetes mellitus, } \\
\text { Plaquetopenia, } \\
\text { Ginecomastia, } \\
\text { Síndrome metabólico }\end{array}$ \\
\hline GBMA & M & 20 & $\mathrm{RM}$ & Si & NR & Hetero & Zurdo & $\begin{array}{l}\text { MFD, ZSD, HPD, RPD, } \\
\text { VPM }\end{array}$ & $\begin{array}{l}\text { Distonías de torsión, } \\
\text { Diabetes mellitus }\end{array}$ \\
\hline BRLM & M & 32 & TOC TCE & $\mathrm{Si}$ & Hiper & Hetero & Diestro & VPM, CBZ, QTP & Somnolencia+Sialorrea \\
\hline ACC & M & 22 & TLP & Si & Normal & Hetero/Auto & Diestro & QTP, VPM, CLZ & Ninguna \\
\hline RLM & $\mathrm{F}$ & 32 & RM+Epilepsia & $\mathrm{Si}$ & Normal & Hetero/Auto & Diestro & VPM, LTC, PRM, QTP & Plaquetopenia \\
\hline CLC & M & 48 & EZF & $\mathrm{Si}$ & NR & Hetero/Auto & Diestro & HPD, QTP, VPM & Distonías de torsión \\
\hline EMZE & M & 59 & EZF & $\mathrm{Si}$ & NR & Hetero/Auto & Diestro & HPD, QTP, CBZ & Distonías de torsión \\
\hline GMA & M & 35 & RM+Autismo & No & Normal & Hetero/Auto & Diestro & CPMZ, CZP, LZP, VPM & Distonías de Torsión \\
\hline
\end{tabular}

TOC: trastorno obsesivo compulsivo; RM: retraso mental; TCE: traumatismo craneoencefálico; EZF: esquizofrenia; QTP: quetiapina; HPD: haloperidol; ZSD: ziprasidona; CBZ: carbamazepina; OLZ: olanzapina; VPM: valproato de mg; CLZ: clonazepam; LTC: levetiracetam; LZP: lorazepam; MFD: metilfenidato; RPD: risperidona; PRD: primidona; CPMZ: clorpromazina; CZP: clozapina.

Tabla 2. Se especifica el sitio aleatorio donde se realizaron los procedimientos. Termocoagulación por radiofrecuencia en los primeros cinco pacientes y el sitio donde posteriormente se realizó una segunda lesión para lograr la reducción de la conducta agresiva

\begin{tabular}{|l|l|l|l|l|l|}
\hline Caso & Dominancia & Primer procedimiento & Segundo procedimiento & Sitio complementario & Blanco final efectivo \\
\hline LSF & Diestro & $\mathrm{AD}+\mathrm{HI}$ & $\mathrm{Al}+\mathrm{HI}$ & $\mathrm{Al}$ & $\mathrm{AD}+\mathrm{Al}$ \\
\hline $\mathrm{ASF}$ & Zurdo & $\mathrm{Al}+\mathrm{HI}$ & $\mathrm{HD}$ & $\mathrm{HD}$ & $\mathrm{HI}+\mathrm{HD}$ \\
\hline $\mathrm{AAJ}$ & Diestro & $\mathrm{AD}+\mathrm{HI}$ & $\mathrm{HD}$ & $\mathrm{HD}$ & $\mathrm{HI}+\mathrm{HD}$ \\
\hline $\mathrm{RCA}$ & Diestro & $\mathrm{AD}+\mathrm{HI}$ & $\mathrm{Al}+\mathrm{HI}$ & $\mathrm{Al}$ & $\mathrm{AD}+\mathrm{Al}$ \\
\hline $\mathrm{NHR}$ & Zurdo & $\mathrm{Al}+\mathrm{HI}$ & $\mathrm{AD}+\mathrm{HD}$ & $\mathrm{AD}+\mathrm{HD}$ & $\mathrm{Al}+\mathrm{AD}$ \\
\hline
\end{tabular}

AD: amígdala derecha; Al: amígdala izquierda; HD: hipotálamo derecho; HI: hipotálamo izquierdo.

sexual fuero muy importantes puesto que en todos los casos hubo casi una desaparición del interés sexual. Ningún paciente posterior a la cirugía requirió manejo con cuidados intensivos (Tabla 4).

\section{Discusión}

Los resultados obtenidos en este trabajo muestran un cambio estadísticamente significativo de la conducta agresiva evaluado por medio de las calificaciones de la OAS. Se apreció una disminución de las medianas entre 13 y 14 puntos a lo largo de 36 meses de seguimiento ( $p$ entre 0.002 y 0.007 ) (Tabla 3). El objetivo principal de este trabajo fue identificar que combinación de lesiones por radiofrecuencia dirigidas al núcleo central de la amígdala o por la lesión del hipotálamo posteromedial pudieran ser más efectivas en producir un cambio clínico. La evaluación de los primeros cinco casos en los que la reaparición de los síntomas del síndrome neuroagresivo casi de inmediato orientó a este grupo de investigadores a considerar la combinación de la amigdalotomía e hipotalamotomía simétricas y contralaterales a la dominancia manual como la primera opción quirúrgica. El trabajo previo de este mismo grupo había vislumbrado esta posibilidad ${ }^{18}$. Así, la principal contribución de 
Tabla 3. En esta tabla se aprecian las calificaciones de la Over Aggression Scale (OAS) en los periodos basales y de seguimiento desde los 6 hasta el máximo seguimiento posible para cada caso, que en 9 pacientes fue de 36 meses. Llama la atención que salvo los dos primeros casos en quienes la mejoría se acentuó después de los 6 meses y posterior a la reintervención, todos los pacientes operados desde el inicio con amigdalotomía e hipotalamotomía contralateral al hemisferio dominante mejoraron desde el inicio. Las diferencias observadas en las medianas de acuerdo con la OAS fueron (prueba de rangos de Wilcoxon): basal vs. 6 meses, -13 puntos ( $p=0.002 ; n=12)$; basal vs. 12 meses, -14 puntos ( $p=0.002 ; n=12)$; basal vs. 18 meses, -13 puntos $(p=$ $0.003 ; n=11)$; basal vs. 24 meses, -14 puntos $(p=0.003 ; n=11)$; basal vs. 30 meses, -13 puntos $(p=0.005 ; n=10)$ y basal vs. 36 meses, -13 puntos $(p=0.007 ; n=9)$

\begin{tabular}{|l|c|c|c|c|c|c|c|}
\hline Caso & Basal & EA 6 & EA 12 & EA 18 & EA 24 & EA 30 & EA 36 \\
\hline LSF & 16 & 9 & 3 & 3 & 3 & 3 & 3 \\
\hline ASF & 16 & 12 & 2 & 3 & 2 & 2 & 2 \\
\hline AAJ & 16 & 3 & 4 & 4 & 4 & 4 & 4 \\
\hline RCA & 16 & 0 & 0 & 0 & 0 & 0 & 0 \\
\hline NHR & 16 & 5 & 4 & 4 & 4 & 4 & 4 \\
\hline GBMA & 16 & 0 & 0 & 0 & 0 & 0 & 0 \\
\hline BRLM & 16 & 5 & 3 & 3 & 3 & 3 & 3 \\
\hline ACC & 16 & 0 & 0 & 0 & 0 & 0 & 0 \\
\hline RLM & 16 & 5 & 4 & 4 & 4 & 4 & 4 \\
\hline CLC & 16 & 0 & 0 & 0 & 0 & 0 & NA \\
\hline EMZE & 16 & 0 & 0 & 0 & 0 & NA & NA \\
\hline GMA & 16 & 0 & 0 & NA & NA & NA & NA \\
\hline Media & 16 & 3.25 & 1.67 & 1.91 & 1.82 & 2.00 & 2.22 \\
\hline DE & 0 & 4.07 & 1.83 & 1.87 & 1.83 & 1.83 & 1.79 \\
\hline DEA & & & & & & & \\
\hline
\end{tabular}

DE: NA: no accesible.

este trabajo ha sido el medir con un instrumento estandarizado los cambios obtenidos por una TCRF de la AC y del HPM en pacientes seguidos por 36 meses y también describir que la agresividad puede tener una lateralidad hemisférica compartida con la dominancia manual.

Otros trabajos previos, como los informados por este mismo grupo en el año 2012 había ya encontrado que la lesión múltiple de las proyecciones del frontal a las estructuras de los núcleos basales puede disminuir la conducta agresiva a costa de disminuir las funciones ejecutivas ${ }^{16}$. Los reportes de todos los autores previos quienes han ya informado sobre la efectividad de la AC y de la HPM no han sido seguidos tan largamente y no han usado un instrumento validado en México ${ }^{10-16}$.

Sin embargo, este trabajo aún es un estudio preliminar que busca una opción quirúrgica en los casos

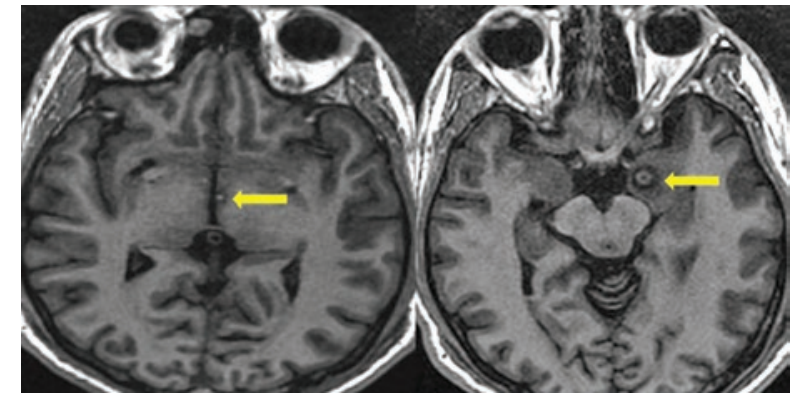

Figura 1. En estas dos imágenes axiales de resonancia magnética en fase $T 1$ se aprecian las señales típicas agudas de las nucleotomías producidas por termocoagulación por radiofrecuencia para la hipotalamotomía posteromedial a la izquierda y la NCA a la derecha (se muestran señalados por flechas amarillas).

de pacientes con agresividad refractaria que no han mejorado en sus condiciones clínicas, familiares y sociales con los tratamientos convencionales. Las principales limitaciones del presente trabajo corresponden a los propios de un estudio piloto autocontrolado y son el reducido número de casos incluidos y la ausencia de un grupo control. Sin embargo, salvo los estudios de Ramamurti ${ }^{12}$, la mayor parte de los artículos publicados son series de casos con un número reducido de pacientes enrolados y sin un grupo control; lo cual no es posible en vista que un procedimiento quirúrgico de este tipo no puede compararse con una simulación de cirugía asignada aleatoriamente.

Aunque se realizó un estudio autocontrolado y se aplicó una prueba estadística no paramétrica (prueba de rangos de Wilcoxon), es necesario extender la experiencia en otros grupos de investigadores dedicados a este campo de la medicina.

El paciente ASF, zurdo, mejoró sus condiciones clínicas al completar la TCRF del hipotálamo derecho, considerando que ya se había hecho una lesión de la amígdala e hipotálamo izquierdos. En este caso particular la combinación de los dos hipotálamos y de la amígdala no dominante mejoraron las condiciones del enfermo y nos orientan a pensar que la evaluación de la dominancia pudiera ser difícil en ocasiones o que las lesiones múltiples, como en el caso de capsulotomía y cingulotomía bilaterales, son también efectivas.

El mecanismo de acción de estos procedimientos quirúrgicos se ha discutido ya en los trabajos de los autores originales que han mencionado acerca de la interrupción del sistema límbico como la principal explicación del mecanismo de acción de estos procedimientos $^{10-13}$.

Este grupo de investigadores considera que sería interesante explorar los efectos de estos mismos núcleos cerebrales mediante estimulación eléctrica 
Tabla 4. Se describen los efectos colaterales de la hipotalamotomía posteromedial y de la amigdalotomía central. Siete de 12 pacientes mostraron efectos secundarios, siendo el de mayor frecuencia la somnolencia. En ningún caso fue definitivo alguno de los efectos colaterales. También se describe el efecto sobre la reducción en la conducta sexual. Cuatro de cinco casos disminuyeron su comportamiento sexual

\begin{tabular}{|c|c|c|c|}
\hline Caso & $\begin{array}{l}\text { Efectos secundarios postoperatorios inmediatos (entre } \\
7 \text { y } 14 \text { días de duración) }\end{array}$ & Sexualidad preoperatoria & $\begin{array}{l}\text { Sexualidad } \\
\text { postoperatoria }\end{array}$ \\
\hline LSF & Somnolencia & $\begin{array}{l}\text { Hipersexualidad } \\
\text { Pederastia }\end{array}$ & $\begin{array}{l}\text { Reducción } \\
\text { Anulación de Pederastia }\end{array}$ \\
\hline ASF & Ninguna & $\begin{array}{l}\text { Hipersexualidad } \\
\text { Laceración anal autoinfligida con objetos }\end{array}$ & Anulación \\
\hline AAJ & Somnolencia & No reportada & No reportada \\
\hline $\mathrm{RCA}$ & Somnolencia & Hipersexualidad & Anulación \\
\hline $\mathrm{NHR}$ & Ninguna & Hipersexualidad & Reducción \\
\hline GBMA & Ninguna & No reportada & No reportada \\
\hline BRLM & Ninguna & Hipersexualidad & Sin cambios \\
\hline ACC & Ninguna & Normal & Sin cambios \\
\hline RLM & Monoparesia braquial, anorexia, somnolencia, hipertermia & Normal & Sin cambios \\
\hline CLC & Somnolencia & No reportada & No reportada \\
\hline EMZE & Somnolencia, Anorexia & No reportada & No reportada \\
\hline GMA & Somnolencia, hipotensión arterial (requirió aminas) & Normal & Reducción \\
\hline
\end{tabular}

cerebral profunda, donde podría ser utilizada la aleatorización y el cegamiento. Al implantar un paciente con electrodos cerebrales, y previo consentimiento informado, los pacientes pueden ser seleccionados al azar para encontrase bajo estimulación o fuera de ella y como la estimulación es silente en cuanto a su acción local también es relativamente fácil diseñar un estudio doble o triple ciego. No obstante, no se ha realizado algún ensayo clínico de ese tipo.

Claramente es necesario que otro grupo de investigadores considere replicar las observaciones sobre la dominancia hemisférica de la conducta agresiva. La dominancia izquierda del lenguaje y de ciertas funciones ejecutivas pudieran orientar sobre la posibilidad de que la conducta agresiva pudiera ser no solo una actividad instintiva sino más bien una actividad motora planeada y ejecutada para lograr un objetivo específico, tal como lo es dirigir la agresión hacia la consecución del territorio, de los satisfactores o del nivel jerárquico.

Sería importante realizar una evaluación de las condiciones genéticas, hormonales y epigenéticas de los pacientes con un síndrome neuroagresivo; es Ilamativo que en la mayoría de los informes de series de caso tratados neuroquirúrgicamente, los sujetos estudiados son hombres. Finalmente, la posibilidad de realizar procedimientos menos invasivos como radiocirugía o estimulación cerebral profunda es hasta el día de hoy un capítulo pendiente que se limita por la factibilidad de conseguir los equipos de neuromodulación.

\section{Conclusiones}

En este estudio se concluye que las lesiones unilaterales simétricas y simultáneas mediante TCRF del núcleo central de la amígdala y del hipotálamo posteromedial contralaterales a la dominancia motora dan el mismo efecto clínico en la reducción de la agresividad patológica que las lesiones bilaterales.

\section{Bibliografía}

1. Faria MA. Violence, mental illness, and the brain - A brief history of psychosurgery: Part 3 - From deep brain stimulation to amygdalotomy for violent behavior, seizures, and pathological aggression in humans. Surg Neurol Int. 2013:4:91.

2. Yudofsky SC, Silver JM, Jackson W, Endicott J, Williams D. The Overt Aggression Scale for the objective rating of verbal and physical aggression. Am J Psychiatry. 1986;143:35-9.

3. Edlinger M, Welte AS, Yalcin-Siedentopf N, Kemmler G, Neymeyer F, Fleischhacker WW, et al. Trends in pharmacological emergency treatment of patients suffering from schizophrenia over a 16-year observation period. Int Clin Psychopharmacol. 2018:33:197-203.

4. Taber KH, Hurley RA, Yudofsky SC. Diagnosis and treatment of neuropsychiatric disorders. Annu Rev Med. 2010;61:121-33.

5. Rojas G P, Arancibia S P, Bravo S V, Varela G S. Diabetes mellitus inducida por olanzapina: Caso clínico. Rev Méd Chile. 2001;129:1183-5.

6. Escobar-Córdoba F, Suárez-Beltrán MF. Abordaje clínico del paciente violento en atención primaria. Universitas Médica. 2011:52:421-30.

7. Jiménez-Ponce F, García-Muñoz L, Carrillo-Ruiz JD. The role of bioethics in the neurosurgical treatment of psychiatric disorders. Rev Med Hosp Gen Mex. 2015;78:47-54. 
8. Yampolsky C, Bendersky D. Cirugía de los trastornos del comportamiento: el estado del arte. Surg Neurol Int. 2014;5:S211-31.

9. Caruso JP, Sheehan JP. Psychosurgery, ethics, and media: a history of Walter Freeman and the lobotomy. Neurosurg Focus. 2017;43:E6.

10. Sano K, Yoshioka M, Ogashiwa M, Ishijima B, Ohye CJS. Postero-medial hypothalamotomy in the treatment of aggressive behaviors. Confin Neurol. 1966;27:164-7.

11. SanoK. Aggressiveness. In: Schaltenbrand G, WalkerAE, editors. Stereotaxy of the human brain. Anatomical, physiological and clinical application. Stuttgart: Georg Thieme Verlag; 1982..617.21.

12. Ramamurthi B. Stereotactic operation in behaviour disorders. Amygdalotomy and hypothalamotomy. En: Personality and neurosurgery. Springer; 1988. pp. 152-7.

13. Narabayashi H, Uno MJS. Long range results of stereotaxic amygdalotomy for behavior disorders. Confin Neurol. 1966;27:168-71.

14. Ledo-Varela MT, Gimenez-Amaya JM, Llamas A. [The amygdaloid complex and its implication in psychiatric disorders]. An Sist Sanit Navar. 2007;30:61-74.
15. Walker AE, Hassler R, Schaltenbrand G, Andy OJ. Stereotaxy of the human brain: anatomical, physiological and clinical applications. Stuttgart: Georg Thieme Verlag; 1982.

16. Jiménez F, Soto JE, Velasco F, Andrade P, Bustamante JJ, Gómez P, et al. Bilateral cingulotomy and anterior capsulotomy applied to patients with aggressiveness. Stereotact Funct Neurosurg. 2012;90:151-160.

17. Franzini A, Broggi G, Cordella R, Dones I, Messina GJWn. Deep-brain stimulation for aggressive and disruptive behavior. 2013:80:S29.

18. Garcia-Munoz L, Carrillo-Ruiz J, Favila-Bojorquez J, Lopez-Valdes J, Jimenez-Ponce FJ. Treatment of refractory aggressiveness by amygdalotomy and posteromedial hypothalamotomy by radiofrequency. 2019;68:91-8.

19. Páez F, Licon E, Fresán A, Apiquian R, Herrera-Estrella M, García-Anaya $\mathrm{M}$, et al. Estudio de validez y confiabilidad de la escala de agresividad explícita en pacientes psiquiátricos. 2002;25:21-6.

20. Oldfield RC. The assessment and analysis of handedness: the Edinburgh inventory. 1971;9:97-113.

21. Talairach JJ, Tournoux P. Co-planar stereotaxic atlas of the human brain-3-dimensional proportional system: An Approach to Cerebral Imaging. 1988;39:145. 